\title{
For Quantum and Reversible Computing, Intervals Are More Appropriate Than General Sets, And Fuzzy Numbers Than General Fuzzy Sets
}

\author{
Oscar Galindo and Vladik Kreinovich \\ Department of Computer Science \\ University of Texas at El Paso, El Paso, TX 79968, USA \\ ogalindomo@miners.utep.edu, vladik@utep.edu
}

\begin{abstract}
Need for faster and faster computing necessitates going down to quantum level - which means involving quantum computing. One of the important features of quantum computing is that it is reversible. Reversibility is also important as a way to decrease processor heating and thus, enable us to place more computing units in the same volume. In this paper, we argue that from this viewpoint, interval uncertainty is more appropriate than the more general set uncertainty and, similarly, that fuzzy numbers (for which all alpha-cuts are intervals) are more appropriate than more general fuzzy sets. We also explain why intervals (and fuzzy numbers) are indeed ubiquitous in applications.
\end{abstract}

Keywords: reversible computing, quantum computing, interval uncertainty, set uncertainty, fuzzy numbers, fuzzy sets

\section{Need for Quantum and Reversible Computing}

Need for quantum computing. Our current computers are very fast in comparison with what was available a few years ago. However, no matter how fast the computers, there are always computational tasks - from bioinformatics, from other disciplines - that necessitate even faster computers.

To speed up computers, we need to be able to squeeze in more and more memory cells and processing cells into the same volume. For that, we need to make these cells as small as possible. Already, the existing cells contain a small number of molecules. If we decrease them further, they will contain a few molecules and therefore, we will need to take into account quantum effects; see, e.g., [4, 23]. This is exactly the domain of quantum computing - computations that take quantum effects into account; see, e.g., [17].

Quantum computing: additional advantages. Known good news about quantum computing is that, in addition to a speed up caused by microminiaturization of the processing units, we achieve an additional speed up by using innovative algorithms specifically designed for quantum computing.

For example, with quantum computers, we can decrease the time needed to find an element in an unsorted array of size $n$ from $n$ to $\sqrt{n}$ computational steps $[6,7,17]$. We can also reduce the time needed to factor large integers of $n$ digits - task needed to decode currently encoded messages - from exponential to polynomial in $n[17,21,22]$.

Need for reversible computing. One challenge in designing quantum computers is that on the quantum level, all equations are time-reversible [4, 17, 23].

Some elementary computer operations are reversible. For example, if we know the original value $x_{1}$ and we know the result $y=x_{1}+x_{2}$ of adding another value $x_{2}$ to $x_{1}$, then we can uniquely reconstruct this another value as $x_{2}=y-x_{1}$.

Other elementary operations, however, are not reversible. For example, even when we know the value $x_{1}=0$ and we know the result $y=x_{1} \cdot x_{2}=0$ of multiplying $x_{1}$ by some other value $x_{2}$, then, based on this information, we cannot uniquely reconstruct $x_{2}$.

Multiplying by zero may be not that common in computer algorithms, but a similar phenomenon occurs for a much more common "and"-operation $a \& b$ : even when we know that $a$ is false, and we know the result (false) of computing $c=a \& b$, we cannot uniquely reconstruct the value $b$. In this case, $b$ could be both true or false.

Similarly, for "or" -operation $a \vee b$ : when we know that $a$ is true, and we know the truth value of $a \vee b$, we 
cannot uniquely reconstruct $b$.

Reversibility is also important because, according to statistical physics, any irreversible process means increasing entropy, and this leads to heat emission; see, e.g., [4, 23]. Already overheating is one of the reasons why we cannot pack too many processing units into the same volume. So, to pack more, it is desirable to reduce this heat emission - e.g., by using only reversible computations.

Comment. The heat-reducing need for reversible computing was first described in [5]; in that paper, reversible computations are also called conservative.

\section{When Are Algorithms for Data Processing Under Uncertainty Reversible}

Need to take uncertainty into account. When are algorithms reversible? We use computers mostly to process data. When processing data, we need to take into account that data comes from measurements, and measurements are never absolutely accurate, the measurement result $\widetilde{x}$ is, in general, somewhat different from the actual value $x$ of the corresponding quantity. It is therefore necessary to take this uncertainty into account when processing data.

Need for interval uncertainty. In many real life situations, the only information that we have about the measurement error $\Delta x \stackrel{\text { def }}{=} \widetilde{x}-x$ is the upper bound $\Delta$ on its absolute value: $|\Delta x| \leq \Delta$. Once we have a measurement result $\widetilde{x}$, then the only information that we can conclude about the actual value $x$ of the corresponding quantity is that this value is somewhere in the interval $[\widetilde{x}-\Delta, \widetilde{x}+\Delta]$. Such interval uncertainty indeed appears in many practical applications; see, e.g., [8, 11, 12, 16, 19].

Data processing under interval uncertainty. In a data processing algorithm, we take several inputs $x_{1}, \ldots, x_{n}$, and we apply an appropriate algorithm to generate the result $y$ depending on these inputs. Let us denote this dependence by $f\left(x_{1}, \ldots, x_{n}\right)$. In situations when, for each input $i$, we only know the interval $X_{i}=$ $\left[\widetilde{x}_{i}-\Delta_{i}, \widetilde{x}_{i}+\Delta_{i}\right]$ of possible values of $x_{i}$, then the only information that we can have about $y$ is that $y$ belongs to the set

$$
\begin{gathered}
Y=f\left(X_{1}, \ldots, X_{n}\right) \\
\stackrel{\text { def }}{=}\left\{f\left(x_{1}, \ldots, x_{n}\right): x_{1} \in X_{1}, \ldots, x_{n} \in X_{n}\right\} .
\end{gathered}
$$

When the sets $X_{i}$ are intervals and the function $f\left(x_{1}, \ldots, x_{n}\right)$ is continuous, the resulting set $Y$ is also an interval.
In most practical situations, the measurement errors are relatively small, so we can expand the function $f\left(x_{1}, \ldots, x_{n}\right)=f\left(\widetilde{x}_{1}-\Delta x_{1}, \ldots, \widetilde{x}_{n}-\Delta x_{n}\right)$ in Taylor series and retain only linear terms. Then, we get

$$
\begin{aligned}
f\left(x_{1}, \ldots, x_{n}\right) & =f\left(\widetilde{x}_{1}-\Delta x_{1}, \ldots, \widetilde{x}_{n}-\Delta x_{n}\right) \\
& \approx \widetilde{y}-\sum_{i=1}^{n} c_{i} \cdot \Delta x_{i},
\end{aligned}
$$

where $\widetilde{y} \stackrel{\text { def }}{=} f\left(\widetilde{x}_{1}, \ldots, \widetilde{x}_{n}\right)$ and $\left.c_{i} \stackrel{\text { def }}{=} \frac{\partial f}{\partial x_{i}}\right|_{x_{i}=\widetilde{x}_{i}}$. In other words, the function $f\left(x_{1}, \ldots, x_{n}\right)$ becomes a linear function of $x_{i}$ :

$f\left(x_{1}, \ldots, x_{n}\right)=\widetilde{y}-\sum_{i=1}^{n} c_{i} \cdot\left(\widetilde{x}_{i}-x_{0}\right)=c_{0}+\sum_{i=1}^{n} c_{i} \cdot x_{i}$,

where $c_{0} \stackrel{\text { def }}{=} \widetilde{y}-\sum_{i=1}^{n} c_{i} \cdot \widetilde{x}_{i}$. In other words, data processing can be, in effect, reduced to two operations: multiplication by a constant $c_{i}$ and addition.

For intervals, both operations can be described explicitly. Indeed, if for the quantity $x$, we only know that $x$ belongs to the interval $X=[x, \bar{x}]$, then the set $Y=c \cdot X=\{c \cdot x: x \in X\}$ takes the following form:

- for $c>0, Y=[c \cdot \underline{x}, c \cdot \bar{x}]$, and

- for $c<0, Y=[c \cdot \bar{x}, c \cdot \underline{x}]$.

For addition, if we know that

$$
x_{1} \in\left[\underline{x}_{1}, \bar{x}_{1}\right] \text { and } x_{2} \in\left[\underline{x}_{2}, \bar{x}_{2}\right] \text {, }
$$

then:

- the smallest value of $y=x_{1}+x_{2}$ is attained when both $x_{1}$ and $x_{2}$ are the smallest and is, thus, equal to $\underline{x}_{1}+\underline{x}_{2}$, and

- the largest value of $y=x_{1}+x_{2}$ is attained when both $x_{1}$ and $x_{2}$ are the largest and is, thus, equal to $\bar{x}_{1}+\bar{x}_{2}$.

Thus, in this case, the interval $Y=[y, \bar{y}]$ has the form

$$
Y=\left[\underline{x}_{1}+\underline{x}_{2}, \bar{x}_{1}+\bar{x}_{2}\right] \text {. }
$$

When is this data processing reversible? Multiplication by a constant is always reversible: indeed, if we know the interval $Y=c \cdot X$, then, as one can easily see, we can reconstruct the original interval $X$ as $X=c^{-1} \cdot Y$, i.e., as the set of all the values $c^{-1} \cdot y$, where $y \in Y=c \cdot X=\{c \cdot x: x \in X\}$. 
Similarly, addition of intervals is also reversible, in the same sense in which addition of real numbers is reversible: if we know the interval $X_{1}=\left[\underline{x}_{1}, \bar{x}_{1}\right]$ and we know the sum $Y=X_{1}+X_{2}=[y, \bar{y}]$ of this interval $X_{1}$ with some other interval $X_{2}$, then, from the formulas $y=\underline{x}_{1}+\underline{x}_{2}$ and $\bar{y}=\bar{x}_{1}+\bar{x}_{2}$, we can reconstruct $X_{2}=\left[\underline{x}_{2}, \bar{x}_{2}\right]$ as $\underline{x}_{2}=\underline{y}-\underline{x}_{1}$ and $\bar{x}_{2}=\bar{y}-\bar{x}_{1}$.

From interval uncertainty to a more general set uncertainty. In some cases, in addition to knowing that values of $x$ are within a certain interval $[\underline{x}, \bar{x}]$, we also know that some values from this interval are not possible. In this case, the set $X$ of possible values of $x$ is different from an interval.

This set must be bounded. No matter how crude the measurements are, there is always an upper bound $\Delta$ on the measurement error. Thus, after each measurement, based on the measurement result $\widetilde{x}$, we can conclude that the set of possible values of the corresponding quantity is located within the interval $[\widetilde{x}-\Delta, \widetilde{x}+\Delta]$ and is, thus, bounded.

It makes sense to consider this set to be closed. In general, we can safely assume that the set $X$ is closed. Indeed, suppose that $x_{0}$ is a limit point of the set, i.e., that for every $\varepsilon>0$, there are elements $x \in X$ is any $\varepsilon$-neighborhood $\left(x_{0}-\varepsilon, x_{0}+\varepsilon\right)$ of this value $x_{0}$.

This means that no matter how accurately we measure the corresponding value, we will not be able to distinguish between the limit value $x_{0}$ and a sufficiently close value $x \in X$. In other words, no matter how accurately we measure, we will never be able to conclude that the value $x_{0}$ is not possible.

So, if we optimistically interpret each not-impossible value as a possible one, it makes sense to conclude that $x_{0}$ is possible. Thus, we can conclude that the set of possible values of each quantity $x$ contains all its limit points, i.e., is closed - since we cannot experimentally distinguish the original set $X$ from its closure.

Comment. In this paper, we consider the maximally optimistic approach, that anything which is not proven to be impossible is actually possible. At first glance, it may also be possible to consider a maximally pessimistic approach - according to which we consider a value to be possible only if we have proven it to be possible, i.e., if we are absolutely sure that this value is possible.

Unfortunately, this maximally pessimistic approach does not lead to any meaningful selection. For example, when the set of observed values is everywhere dense on the interval $[0,1]$, with this maximally pessimistic approach, there is no value $v \in[0,1]$ which is absolutely possible: we can always have the set of all possible values to be equal $[0, v) \cup(v, 1]$; this will be consistent with all the measurement results.

Data processing under set uncertainty. If we know the set $X_{1}$ of possible values of a quantity $x_{1}$, and we know the set $X_{2}$ of possible values of a quantity $x_{2}$, then the set $Y \stackrel{\text { def }}{=} X_{1}+X_{2}$ of possible values of the sum $y=x_{1}+x_{2}$ is equal to

$$
Y=\left\{x_{1}+x_{2}: x_{1} \in X_{1} \text { and } x_{2} \in X_{2}\right\} .
$$

When is this operation reversible? As we have mentioned earlier, for numbers, addition is reversible in the sense that if we know $x_{1}$ and we know the sum $x_{1}+x_{2}$, then we can uniquely reconstruct the value $x_{2}$. Similarly, addition is reversible for intervals. A natural question is: when is addition reversible for sets? In other words, when from the sets $X_{1}$ and $Y=X_{1}+X_{2}$, we can uniquely reconstruct the set $X_{2}$ ?

Known result: addition is reversible only for intervals. We have already mentioned that addition is reversible for intervals. Interestingly (see, e.g., [2, 3]), addition is reversible only for intervals: namely, if we add any non-interval bounded closed set $S$ to the class of all intervals, additions stops being reversible.

The proof of this result is very straightforward: if we take $\underline{S} \stackrel{\text { def }}{=} \inf \{x: x \in S\}$ and $\bar{S} \stackrel{\text { def }}{=} \sup \{x: x \in S\}$, then we have

$$
[\underline{S}, \bar{S}]+[\underline{S}, \bar{S}]=[\underline{S}, \bar{S}]+S(=[2 \underline{S}, 2 \bar{S}]),
$$

but $[\underline{S}, \bar{S}] \neq S$.

Case of fuzzy uncertainty. In many real-life situations, in addition to the guaranteed upper bound $\Delta$ on the absolute value of the measurement error, experts usually know that most probably (or, to be precise, with some high degree of certainty $\beta$ ) measurement errors can be bounded by a smaller bound $\Delta(\beta)<\Delta$. As a result, in addition to the interval $[\widetilde{x}-\Delta, \widetilde{x}+\Delta]$ that is guaranteed to contain the actual value with $100 \%$ confidence, we have several narrower intervals $[\widetilde{x}-\Delta(\beta), \widetilde{x}+\Delta(\beta)]$ that contain the actual value $x$ with the corresponding confidences $\beta$. In other words, we have a nested family of intervals corresponding to different values $\beta$ : the larger the $\beta$ (i.e., the higher the desired confidence), the wider the interval.

Such a family of nested interval is, in effect, an equivalent way of representing a fuzzy number; see, e.g., $[1,9,13,14,15,18,24]$. If instead of intervals, we have more general sets $S(\beta)$, then we have a fuzzy set. The corresponding sets $S(\beta)$ (in particular, intervals) 
are known as $\alpha$-cuts of the nested-family fuzzy set, where $\alpha \stackrel{\text { def }}{=} 1-\beta$.

For such fuzzy sets, we can define operations layer-bylayer: for each $\beta$ (i.e., equivalently, for each $\alpha$ ), we process all the sets (or intervals) corresponding to this value $\beta$. This is equivalent to what Zadeh proposed for processing fuzzy numbers (and, more generally, fuzzy sets) and what is now known as Zadeh's extension principle. This principle is still the most widely used approach to processing fuzzy numbers and fuzzy sets.

Since fuzzy numbers correspond to intervals, and general fuzzy sets to general sets, we arrive at the same conclusion as for sets and intervals [2, 3]: addition is reversible only for fuzzy numbers; if we add any fuzzy set which is not a fuzzy number to the class of all fuzzy numbers, additions stops being reversible.

\section{Good News: Intervals and Fuzzy Numbers Are Ubiquitous - An Explanation}

Intervals are ubiquitous. In the previous section, we showed that intervals (and fuzzy numbers) are preferable since they lead to reversible data processing. Interestingly, intervals (and fuzzy numbers) are indeed ubiquitous, they occur much much more frequently in practice as descriptions of uncertainty than any other sets; see, e.g., [19]. Why is that?

A possible explanation: main idea. To understand why intervals are ubiquitous in non-probabilistic uncertainty, let us recall why in probabilistic uncertainty, the most frequently used distributions - normal (Gaussian) ones - are ubiquitous. The usual explanation is that usually, there are many different independent sources of measurement error. As a result, the measurement error is a sum of a large number of small independent random variables each of which corresponds to one of these sources. It is known that in the limit, when the number of terms in such a sum increases, the distribution of the sum tends to normal; this is known as the Central Limit Theorem; see, e.g., [20]. This limit result means that when the number of components is large, the corresponding definition is close to normal. Thus, from the practical viewpoint, we can safely consider the distribution to be normal.

In non-probabilistic case too, the measurement error is the sum of a large number $n$ of small independent error components:

$$
\Delta x=\Delta x^{(1)}+\Delta x^{(2)}+\ldots+\Delta x^{(n)} .
$$

So, if for each of the components $\Delta x^{(k)}$, we know the set $X^{(k)}$ of possible values, then the set $S$ of possible values of their sum is equal to the sum of these sets:

$$
\begin{gathered}
X=X^{(1)}+\ldots+X^{(n)} \\
=\left\{\Delta x^{(1)}+\Delta x^{(2)}+\ldots+\Delta x^{(n)}:\right. \\
\left.\Delta x^{(1)} \in X^{(1)}, \ldots, \Delta x^{(n)} \in X^{(n)}\right\} .
\end{gathered}
$$

It can be shown that, under reasonable conditions, when the number of components increases, the resulting set $X$ also tends to an interval; see, e.g., [10].

Need for a more detailed explanation. The asymptotic closeness is good, but for practical applications, it is desirable to know exactly how close is the resulting set $X$ to an interval. This is what we will analyze in this section.

What does closeness of sets mean: a brief reminder. For every positive real number $\varepsilon>0$, two points $a$ and $b$ are $\varepsilon$-close is $|a-b| \leq \varepsilon$. It is therefore reasonable to say that the sets $A$ and $B$ are $\varepsilon$-close if:

- every point $a \in A$ is $\varepsilon$-close to some point $b \in B$, and

- every point $b \in B$ is $\varepsilon$-close to some point $a \in A$.

The smallest value $\varepsilon$ with this property is known as the Hausdorff distance $d_{H}(A, B)$ between the two sets.

How to measure smallness of a closed set. The size of a closed set $A$ can be naturally measured by its diameter $\operatorname{diam}(A)$, i.e., the largest possible distance $d\left(a, a^{\prime}\right)$ between the two points $a, a^{\prime}$ from this set. For bounded closed subsets $A$ of a real line, the diameter is simply equal to the difference between its largest point $\sup A$ and $\inf A: \operatorname{diam}(A)=\sup A-\inf A$.

Our main result. Now, we are ready to formulate our main result.

Proposition 1. If $\operatorname{diam}\left(A_{i}\right) \leq \varepsilon$ for all $i=1, \ldots, n$, then for the sum $A=A_{1}+\ldots+A_{n}$, its Hausdorff distance from some interval $I$ does not exceed $\varepsilon / 2$ :

$$
d_{H}(A, I) \leq \varepsilon / 2 .
$$

Comment. This bound cannot be improved, as shown by the following result:

Proposition 2. For every $n$, there exist closed bounded sets $A_{1}, \ldots, A_{n}$ for which $\operatorname{diam}\left(A_{i}\right) \leq \varepsilon$ for all $i$, and for whose sum $A=A_{1}+\ldots+A_{n}$, for every interval $I$, we have $d_{H}(A, I) \geq \varepsilon / 2$.

Proof of Proposition 1. Let us show that the desired inequality holds for the interval $[\underline{a}, \bar{a}]$, where: 
- $\underline{a} \stackrel{\text { def }}{=} \underline{a}_{1}+\ldots+\underline{a}_{n}$, where $\underline{a}_{i} \stackrel{\text { def }}{=} \inf A_{i}$, and

- $\bar{a} \stackrel{\text { def }}{=} \bar{a}_{1}+\ldots+\bar{a}_{n}$, where $\bar{a}_{i} \stackrel{\text { def }}{=} \sup A_{i}$.

To prove the desired inequality, we need to show that:

- every point $a \in A$ is $(\varepsilon / 2)$-close to some point from the interval $I=[\underline{a}, \bar{a}]$, and

- vice versa, that every point $b$ from the interval $I=[\underline{a}, \bar{a}]$ is $(\varepsilon / 2)$-close to some point from the sum set $A$.

Let us first prove that every point $a \in A$ is $(\varepsilon / 2)$-close to some point from the interval $I=[\underline{a}, \bar{a}]$. Indeed, by definition of the sum set $A$, every point $a$ from this set has the form $a=a_{1}+\ldots+a_{n}$, where $a_{i} \in A_{i}$ for all $i$. Every point $a_{i} \in A_{i}$ is bounded by this set's inf and sup:

$$
\underline{a}_{i}=\inf A_{i} \leq a_{i} \leq \sup A_{i} \leq \bar{a}_{i} .
$$

By adding up $n$ such inequalities, and taking into account that:

- $\underline{a}=\underline{a}_{1}+\ldots+\underline{a}_{n}$,

- $a=a_{1}+\ldots+a_{n}$, and

- $\bar{a}=\bar{a}_{1}+\ldots+\bar{a}_{n}$,

we conclude that $\underline{a} \leq a \leq \bar{a}$, i.e., that the value $a$ actually itself belongs to the interval $I$. So, in this case, we can take $b=a$, and get $|a-b|=0 \leq \varepsilon / 2$.

Let us prove that, vice versa, every point $b$ from the interval $I$ is $(\varepsilon / 2)$-close to some point $a \in A$. Indeed, since all $A_{i}$ are closed sets, they contain their limit points $\underline{a}_{i}=\inf A_{i} \in A_{i}$. Thus, $\underline{a}=\underline{a}_{1}+\ldots+\underline{a}_{n} \in A$.

Since $b \in I$, we have $b \geq \underline{a}$, so $b$ is greater than or equal to some point $a \in \bar{A}$. Let us define

$$
a_{0}=\sup \{a \in A: a \leq b\} .
$$

Since all $A_{i}$ are closed sets, the sum $A$ of these sets is also closed, so $a_{0}$, as a limit of elements from $A$, also belongs to $A$. Of course, in the limit, from $a \leq b$, we conclude that $a_{0} \leq b$.

If $a_{0}=\bar{a}$, then, from the fact that $a_{0} \leq b \leq \bar{a}$, we conclude that $b=a_{0}=\bar{a}$ and thus, $\left|a_{0}-b\right|=0 \leq \varepsilon / 2$.

Let us now consider the remaining case when

$$
a_{0}<\bar{a}=\bar{a}_{1}+\ldots+\bar{a}_{n} .
$$

Since the point $a_{0}$ is in $A$, it means that

$$
a_{0}=a_{1}+\ldots+a_{n}
$$

for some values $a_{i} \in A_{i}$. For each $i$, we have $a_{i} \leq$ $\sup A_{i}=\bar{a}_{i}$. The inequality $a_{0}<\bar{a}$ implies that we cannot have $a_{i}=\bar{a}_{i}$ for all $i$ : otherwise, we would have

$$
a_{0}=a_{1}+\ldots+a_{n}=\bar{a}_{1}+\ldots+\bar{a}_{n}=\bar{a} .
$$

Thus, there exists an $i$ for which $a_{i}<\bar{a}_{i}$. Let us denote one such index by $i_{0}$; then $a_{i_{0}}<\bar{a}_{i_{0}}$.

Let us now consider a new point $\bar{a}_{0} \in A$ in forming which we replace $a_{i_{0}}$ with $\bar{a}_{i_{0}}$ :

$$
\bar{a}_{0}=a_{1}+\ldots+a_{i_{0}-1}+\bar{a}_{i_{0}}+a_{i_{0}+1}+\ldots+a_{n} .
$$

Here, we have $\bar{a}_{0}-a_{0}=\bar{a}_{i_{0}}-a_{i_{0}}$ and thus, by the definition of the diameter, this difference is smaller than or equal to the diameter $\operatorname{diam}\left(A_{i_{0}}\right)$, which is, in turn, smaller than or equal to $\varepsilon$. Thus,

$$
\left|\bar{a}_{0}-a_{0}\right| \leq \varepsilon
$$

Since $a_{0}$ is the largest point from the set $A$ which is smaller than or equal to $b$, and $\bar{a}_{0}>a_{0}$, we thus conclude that $a_{0} \not \leq b$, i.e., that $b<\bar{a}_{0}$. So, we have $a_{0} \leq b<\bar{a}_{0}$. Here, the sum of the distances $\left|b-a_{0}\right|$ and $\left|b-\bar{a}_{0}\right|$ is equal to $\left|\bar{a}_{0}-a_{0}\right|$ and is, thus, smaller than or equal to $\varepsilon$ : $\left|b-a_{0}\right|+\left|b-\bar{a}_{0}\right| \leq \varepsilon$. Thus, at least one of these two distances must be smaller than or equal to $\varepsilon / 2$ (since otherwise, if they were both greater than $\varepsilon / 2$, their sum would be greater than $\varepsilon$ ).

In each of these two cases, we have a point from the set $A$ (either $a_{0}$ or $\left.\bar{a}_{0}\right)$ which is $(\varepsilon / 2)$-close to the given point $b \in I$. The proposition is proven.

Proof of Proposition 2. Let us take

$$
A_{1}=\ldots=A_{n}=\{0, \varepsilon\} .
$$

Then, as one can easily see,

$$
A=A_{1}+\ldots+A_{n}=\{0, \varepsilon, 2 \varepsilon, \ldots, n \cdot \varepsilon\} .
$$

Let us show, by reduction to a contradiction, that we cannot have $d_{H}(A, I)<\varepsilon / 2$ for any interval $I$.

Indeed, suppose that such an interval exists. Then, by definition of the Hausdorff distance, for the point $0 \in A$, there exists a point $b_{1} \in I$ for which

$$
\left|b_{1}-0\right|=\left|b_{1}\right| \leq d_{H}(A, I) .
$$

Then, since $b_{1} \leq\left|b_{1}\right|$, we have $b_{1} \leq d_{H}(A, I)$. Since $d_{H}(A, I)<\varepsilon / 2$, we thus have $b_{1}<\varepsilon / 2$.

Similarly, for the point $\varepsilon \in A$, there exists a point $b_{2} \in I$ for which $\left|\varepsilon-b_{2}\right| \leq d_{H}(A, I)$ and thus, $\varepsilon-b_{2} \leq$ $d_{H}(A, I)$ and $\varepsilon-d_{H}(A, I) \leq b_{2}$. Since $d_{H}(A, I)<\varepsilon / 2$, we thus have $b_{2}>\varepsilon-\varepsilon / 2=\varepsilon / 2$. 
Since the interval $I$ contains two points $b_{1}<\varepsilon / 2$ and $b_{2}>\varepsilon / 2$, it contains all the points in between, including the point $b=\varepsilon / 2$. However, for this point $b \in I$, the closest points from $A$ are the points 0 and $\varepsilon$ for both of which the distance to $b=\varepsilon / 2$ is equal to $\varepsilon / 2$ and is, thus, greater than $d_{H}(A, I)$ - which contradicts to the definition of Hausdorff distance, according to which every point $b \in I$ must be $d_{H}(A, I)$-close to some point from the set $A$.

This contradiction proves that the inequality $d_{H}(A, I)<\varepsilon / 2$ is impossible and thus, indeed, always $d_{H}(A, I) \geq \varepsilon / 2$. The proposition is proven.

\section{Acknowledgments}

This work was partially supported by the US National Science Foundation via grant HRD-1242122 (CyberShARE Center of Excellence).

The authors are thankful to the anonymous referees for valuable suggestions.

\section{References}

[1] R. Belohlavek, J. W. Dauben, and G. J. Klir, Fuzzy Logic and Mathematics: A Historical Perspective, Oxford University Press, New York, 2017.

[2] B. Bouchon-Meunier, O. Kosheleva, V. Kreinovich, and H. T. Nguyen, "Fuzzy numbers are the only fuzzy sets that keep invertible operations invertible", Proceedings of the International Conference on Information Processing and Management of Uncertainty in Knowledge-Based Systems (IPMU'96), Granada, Spain, July 1-5, 1996, Vol. 2, pp. 1049-1054.

[3] B. Bouchon-Meunier, O. Kosheleva, V. Kreinovich, and H. T. Nguyen, "Fuzzy numbers are the only fuzzy sets that keep invertible operations invertible", Fuzzy Sets and Systems, 1997, Vol. 91, No. 2, pp. 155-163.

[4] R. Feynman, R. Leighton, and M. Sands, The Feynman Lectures on Physics, Addison Wesley, Boston, Massachusetts, 2005.

[5] E. Fredkin and T. Toffoli, "Conservative logic", International Journal of Theoretical Physics, 1982, Vol. 21, pp. 219-253.

[6] L. K. Grover, "A fast quantum mechanical algorithm for database search", Proceedings of the 28th ACM Symposium on Theory of Computing, 1996, pp. 212-219.
[7] L. K. Grover, "Quantum mechanics helps in searching for a needle in a haystack", Physical Reviews Letters, 1997, Vol. 79, No. 2, pp. 325328.

[8] L. Jaulin, M. Kiefer, O. Didrit, and E. Walter, Applied Interval Analysis, with Examples in Parameter and State Estimation, Robust Control, and Robotics, Springer, London, 2001.

[9] G. Klir and B. Yuan, Fuzzy Sets and Fuzzy Logic, Prentice Hall, Upper Saddle River, New Jersey, 1995.

[10] V. Kreinovich, "Why intervals? A simple limit theorem that is similar to limit theorems from statistics," Reliable Computing, 1995, Vol. 1, No. 1, pp. 33-40.

[11] V. Kreinovich, A. Lakeyev, J. Rohn, and P. Kahl, Computational Complexity and Feasibility of Data Processing and Interval Computations, Kluwer, Dordrecht, 1998.

[12] G. Mayer, Interval Analysis and Automatic Result Verification, de Gruyter, Berlin, 2017.

[13] J. M. Mendel, Uncertain Rule-Based Fuzzy Systems: Introduction and New Directions, Springer, Cham, Switzerland, 2017.

[14] H. T. Nguyen and V. Kreinovich, "Nested intervals and sets: concepts, relations to fuzzy sets, and applications", In: R. B. Kearfott and V. Kreinovich (eds.), Applications of Interval Computations, Kluwer, Dordrecht, 1996, pp. 245290.

[15] H. T. Nguyen, C. Walker, and E. A. Walker, A First Course in Fuzzy Logic, Chapman and Hall/CRC, Boca Raton, Florida, 2019.

[16] R. E. Moore, R. B. Kearfott, and M. J. Cloud, Introduction to Interval Analysis, SIAM, Philadelphia, 2009.

[17] M. Nielsen and I. Chuang, Quantum Computation and Quantum Information, Cambridge University Press, Cambridge, 2000.

[18] V. Novák, I. Perfilieva, and J. Močkoř, Mathematical Principles of Fuzzy Logic, Kluwer, Boston, Dordrecht, 1999.

[19] S. G. Rabinovich, Measurement Errors and Uncertainties: Theory and Practice, Springer, New York, 2005.

[20] D. J. Sheskin, Handbook of Parametric and Nonparametric Statistical Procedures, Chapman and Hall/CRC, Boca Raton, Florida, 2011. 
[21] P. Shor, "Polynomial-time algorithms for prime factorization and discrete logarithms on a quantum computer", Proceedings of the 35th Annual Symposium on Foundations of Computer Science, Santa Fe, New Mexico, November 20-22, 1994.

[22] P. Shor, "Polynomial-time algorithms for prime factorization and discrete logarithms on a quantum computer", SIAM J. Sci. Statist. Comput., 1997, Vol. 26, pp. 1484-1509.
[23] K. S. Thorne and R. D. Blandford, Modern Classical Physics: Optics, Fluids, Plasmas, Elasticity, Relativity, and Statistical Physics, Princeton University Press, Princeton, New Jersey, 2017.

[24] L. A. Zadeh, "Fuzzy sets", Information and Control, 1965, Vol. 8, pp. 338-353. 